\title{
Modeling of Poly-Silicon Carrier Transport with Explicit Treatment of Grains and Grain Boundaries
}

\author{
EDWIN C. KAN ${ }^{\mathrm{a}}$ and ROBERT W. DUTTON ${ }^{\mathrm{b}}$ \\ a 404 Phillips Hall, Cornell University, Ithaca, NY 14853; \\ ${ }^{\mathrm{b}}$ CIS-X 333, Stanford University, Stanford, CA 94305-4075
}

\begin{abstract}
Explicit treatment of grains and grain boundaries is necessary to model the carrier transport in poly-silicon devices whose feature size is comparable to the grain size. The grain boundaries were modeled by interface traps, and comparison was made between thermionic and diffusion transport across the grain boundaries. It was found that the numerical model for diffusion transport with total trap conservation in grain boundary areas is not physically convergent and shows a strong grid sensitivity. Effects of the critical doping level and the lattice temperature are demonstrated on poly-silicon resistors with 1-D bamboo-type and 2-D realistic microstructures.
\end{abstract}

Keywords: Microstructure, poly-crystalline silicon, carrier transport, device simulation, interface trap

Poly-silicon devices such as TFT for SRAM, LCD, and image sensor, and high-value resistance in analog circuit, have recently received much attention in consideration with its good integration capability with standard CMOS technology [1]. The carrier transport in poly-Si is conventionally modeled like a uniform $\mathrm{Si}$ material with distributed trap density in the band gap [2] related to the average grain size, since more than tens of grains usually exist within the device area of interest [1]. However, with the aggressive scaling of VLSI technology and recent use of laser annealing [3], TFT for SRAM and high resistance poly-Si resistors may have only a few grains within the device, and the device behavior will be heavily dependent on the process conditions owing to the microstructure effects [4]. Nonuniform and anisotropic phenomena from the limited number of grains within the active device area may be important and explicit treatment of carrier transport within grains and across grain boundaries will be necessary. Physical position and size of grains in 2-D can be obtained by AFM (atomic force microscopy) and EBIC (electron-beam induced current) [5] instrumentation.

If the grain size in poly-Si is still larger than $100 \AA$, deviation from single-crystal Si transport (based on the periodic potential approximation) within grains may be negligible. Bandtail transport [4] within grains can be used as the next level of approximation. The grain boundaries in silicon are usually an interface between different crystal orientations and are therefore atomically thin. It is impractical and unphysical to assign nontrivial regions on the order of $50 \AA$ [3] to model the carrier transport across grain boundaries, since the drift-diffusion model is doubtful for that geometrical scale, and gridding for multi-dimension cases 
requires a large number of grid points. Alternatively, the grain boundary can be modeled by interface traps (no physical size assigned to grain boundaries):

$$
\begin{aligned}
\nabla(\varepsilon \nabla \psi)=q\left(n-p-N_{D}^{+}+N_{A}^{-}-N_{D D}^{+}+N_{A A}^{-}\right) \\
\frac{\partial N_{D D}^{+}}{\partial t}=\left(C_{p n} p+e_{n n}\right)\left(N_{D D}-N_{D D}^{+}\right) \\
-\left(C_{n n} n+e_{p n}\right) N_{D D}^{+} \\
\frac{\partial N_{A A}^{-}}{\partial t}=\left(C_{n p} n+e_{p p}\right)\left(N_{A A}-N_{A A}^{-}\right) \\
-\left(C_{p p} p+e_{n p}\right) N_{A A}^{-}
\end{aligned}
$$

where $\varepsilon$ is the dielectric constant, $\psi$ is the potential, $n$ and $p$ are the electron and hole concentrations, $N_{D}^{+}$and $N_{A}^{-}$are non-trapping ionized dopants, and $N_{D D}^{+}$and $N_{A A}^{-}$are donor- and acceptor-state traps. The effect of traps are calculated in rate equations (2) and (3), and then fed back to the Poisson equation (1). The transport equations for $n$ and $p$ follow the conventional forms. The interface trap density is mapped into the discretization scheme of (1) by multiplying the half mesh length of the closest cell to convert into volume trap density. Carrier transport across the grain boundary can be modeled by either thermionic emission or diffusion. The former approach has been taken in [7], although a Monte Carlo simulations is used. The drift-diffusion model is much more computationally efficient and numerically stable, and thus we have chosen to implement the grain boundary treatment under the driftdiffusion model. The thermionic emission model assumes two distinct distribution function at either side of the barrier, and the carrier flux is estimated by the population which has energy over the barrier height. The barrier height is corrected to account for image force and field emission (tunneling), but no scattering event is considered at the barrier. The diffusion transport across the grain boundaries is similar to the case of delta- doped traps, i.e., transport is affected by the barrier height, but scattering mechanisms are homogeneously treated. Detailed model description can be found in classical textbook such as [8].

A poly-Si resistor with 1-D bamboo-like microstructure will be used as the first example for explicit treatment of grain boundaries. Three onemicron-long grains and two grain boundaries are used. The grain boundary is modeled by surface trap concentration $N_{S}$ of $10^{12} \mathrm{~cm}^{-2}$. The critical doping level $N_{T}$ [1] will be $N_{S} / \lambda=10^{16} \mathrm{~cm}^{-3}$, where $\lambda$ is the average grain size. When the grain is doped with less than $N_{T}$, the grain will be fully depleted and the conduction band will have no flat region. The barrier height will reach a maximum when the doping level is close to $N_{T}$. When the doping level exceeds $N_{T}$, most of the traps are filled, the barrier height starts decreasing, and charge neutral regions (flat conduction band) grow from the center of grain toward the grain boundary. This can be clearly observed in both Figures 1 and 2, where thermionic (1a) and diffusion (1b) transport have been demonstrated respectively. However, when diffusion transport is used and the total number of traps is conserved in the delta-doped representation, the barrier height is strongly dependent on grid spacing since the depletion width does not vary with $N_{D}^{-1}$, but with $N_{D}^{-0.5}$ for abrupt junctions. This indicates serious deficiency of using the combination of interface traps and diffusion model, since their physical concepts of interface condition conflict each other. On the other hand, when thermionic transport is used, the barrier height shows no grid dependence for $10 \AA$ to $100 \AA$ minimal grid spacing, (Fig. 2).

As shwon in Figure 3(a), the lattice temperature effect can also be well modeled by the thermionic transport as compared to the experimental results in [1]. When the barrier height is large, the resistivity is much more sensitive to the lattice temperature $T_{L}$, since the thermionic current is proportional to $T_{L}^{2} \exp \left(-E_{B} / k T_{L}\right)$, which is the limiting factor in the resistor studied here. In Figures $3(b-d)$ where the minimal grid spacings are $100 \AA, 50 \AA$ and $10 \AA$, the diffusion transport 

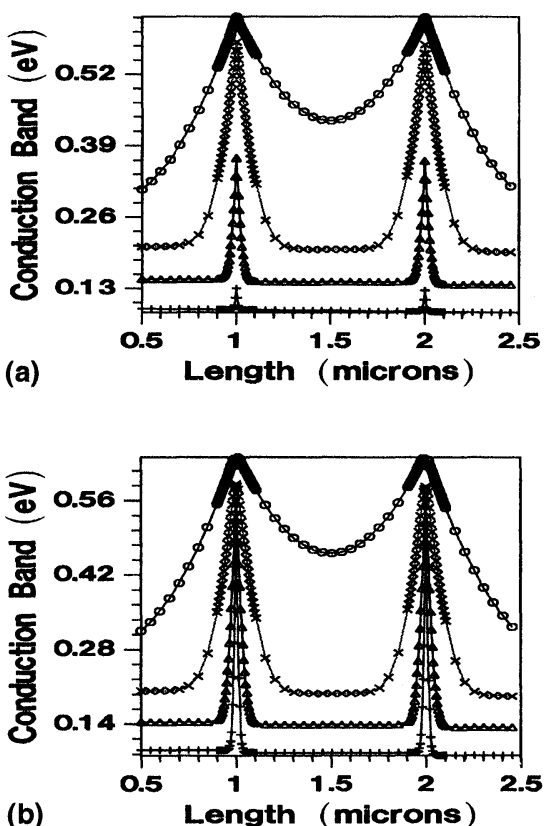

FIGURE 1 Conduction band for various doping levels. (a) by thermionic emission and (b) by diffusion. The critical doping level is around $10^{16} \mathrm{~cm}^{-3}$.

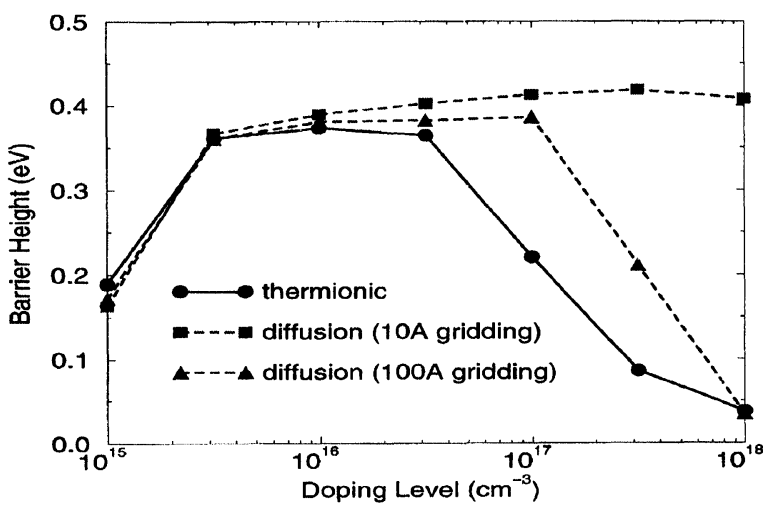

FIGURE 2 Grid dependence of barrier height when diffusion transport across grain boundaries is used. The thermionic model shows no grid dependence for minimal gridding from $100 \AA$ to $10 \AA$.

shows not only strong grid dependence, but also inaccurate resistivity transition when the doping level is in the vicinity of $N_{T}$. It can be observed that the resistivity has less doping-level dependence owing to the estimation of barrier height, and the slope of resistivity with respect to $1 / T_{L}$ does not show a gradual change for doping level across $N_{T}$.

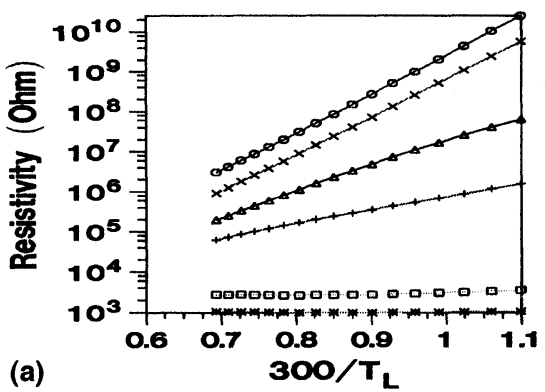

$-1.00+16$

- $3.00+10$

$-7.00+10$

$+1.00+17$

$-1.00+18$

(a)
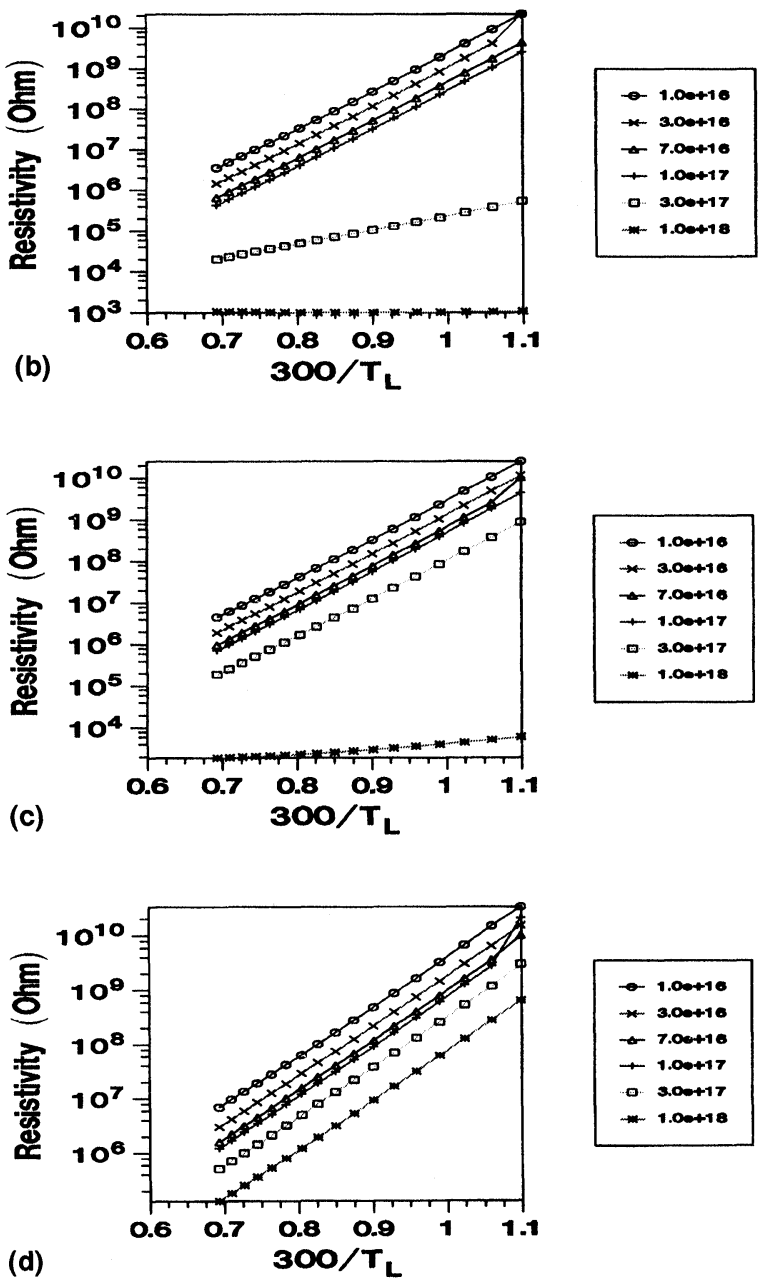

FIGURE 3 The lattice temperature effects on poly-Si resistivity. (a) uses thermionic emission, (b), (c) and (d) use diffusion transport with minimal grid spacing of $100 \AA$, $50 \AA$ and $10 \AA$ respectively.

A poly-Si resistor with microstructure similar to that obtained from EBIC measurement [5] is shown in Figure 4. Thermionic emission transport 
has been used since grid spacing is much harder to control for arbitrary 2-D cases. It can be seen that the current flow pattern is strongly affected by the grain boundaries when the doping level is smaller than $N_{T}$, and less affected when the doping level is larger than $N_{T}$ owing to the barrier height. Stronger depletion can be observed at the grain boundary corners. The lattice temperature effect also shows more complicated behavior in Figure 5, which apparently can not be predicted by simple analytical models [1]. This can be explained by the dependence of 2-D current flow pattern on lattice temperature and barrier height. Detailed analyses are limited by the length of this paper, and will be included in future publications.

We have reported results for carrier transport analyses with explicit treatment of grains and grain boundaries. As the number and orientation of grains become influential for device electrical behaviors, our approach shows good promises for predictive simulation.
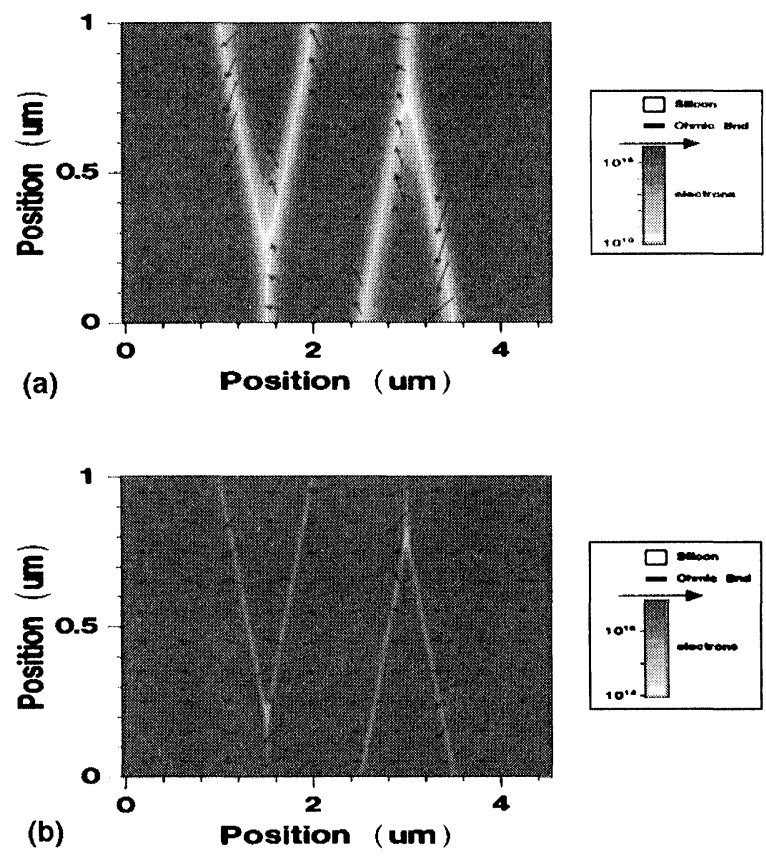

FIGURE 4 Electron concentration and current flow pattern of a 2-D poly-Si resistor. The grain boundaries can be clearly observed at the depletion region. (a) is doped with $10^{16} \mathrm{~cm}^{-3}$ and (b) with $10^{17} \mathrm{~cm}^{-3}$.

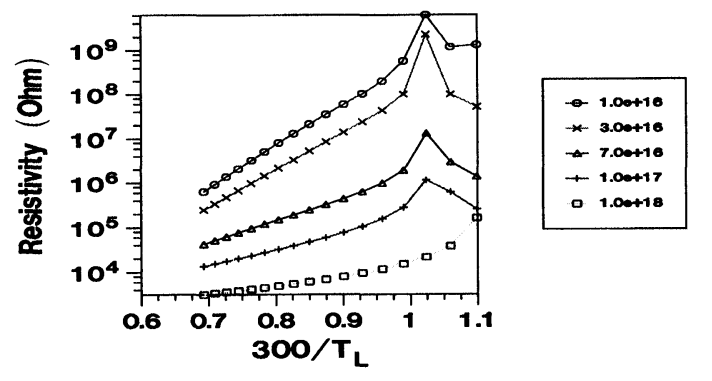

FIGURE 5 The lattice temperature effects on poly-Si resistivity with a $2-\mathrm{D}$ microstructure.

\section{Acknowledgements}

This work was supported in part by DARPA under contracts DABT63-93-C-0053 and F3060296-2-0308.

\section{References}

[1] Kamins, T. (1988). Polycrystalline Silicon for Integrated Circuit Applications, Kluwer Academic.

[2] Hack, M. and Shaw, J. G. "Numerical simulations of amorphous and polycrystalline silicon thin-fim transistor", Proc. 22nd Int. Conf. Solid-State Devices and Materials sendai, Japan, 1990, pp. 999-1003.

[3] Armstrong, G. A., Uppal, S., Brotherton, S. D. and Ayres, J. R. "Modeling of laser-annealed polysilicon TFT characteristics", IEEE Electron Dev. Lett., 18(7), 315318, July 1997.

[4] Pennell, R. and Foerstner, J. "Resistivity control of boron-doped polysilicon resistors", J. Electrochem. Soc., 138(3), 860, March 1991.

[5] Wu, K., Dutton, R. W. and Johnson, N. M. "EBIC measurement and grain boundary recombination in SOI polycrystalline silicon", IEEE Trans. Electron Devices, ED-33(7), 1020, July 1986.

[6] Serikawa, T. et al. Proc. of Intl. Conf. Solid State Dev. and Mat., Osaka, 1995, p. 662.

[7] Shimatani, T. and Koyanagi, M. (1995). "New Monte Carlo simulation for polycrystalline silicon thin-film transistor", IEDM Tech. Dig., p. 297.

[8] Sze, S. M. Physics of Semiconductor Devices, 2nd ed., Wiley, 1981.

\section{Author's Biographies}

Edwin C. Kan received his B.S. degree from National Taiwan University in 1984 and M.S. and $\mathrm{Ph} . \mathrm{D}$. degrees from University of Illinois at Urbana-Champaign in 1988 and 1992, respectively, all in electrical engineering. From 1984 to 1986, he 
served as a second lieutenant in Air Force, Taiwan, Republic of China. From January 1992, he had been with Dawn Technologies as the principal developer of advanced electronic and optical device simulators and technology CAD framework. He has been with Stanford University as a research associate since October 1993, leading projects such as TCAD 1-2-3D tool development, software architecture definition, model hierarchy and MEMS modeling. He joined Cornell University in July 1997 as an assistant professor of School of Electrical Engineering. His main research areas include VLSI technology, semiconductor device physics, composite CAD development, and numerical methods for PDE and ODE.

Robert W. Dutton is Professor of Electrical Engineering at Stanford University and Director of Research in the Center for Intergrated Systems. $\mathrm{He}$ received the B.S., M.S., and Ph.D. degrees from the University of California, Berkeley, in 1966, 1967 and 1970, respectively. He has held summer staff positions at Fairchild, Bell Telephone Laboratories, Hewlett-Packard, IBM Research, and Matsushita during 1967, 1973, 1975, 1977 and 1988 respectively. His research interests focus on Integrated Circuit process, device, and circuit technologies-especially the use of Computer-Aided Design (CAD) and parallel computational methods. Dr. Dutton has published more than 200 journal articles and graduated more than four dozen doctorate students. He was Editor of the IEEE CAD Journal (1984-1986), winner of the 1987 IEEE J. J. Ebers Award, 1988 Guggenheim Fellowship to study in Japan and was elected to the National Academy of Engineering in 1991. In December 1996, Dr. Dutton received the Jack A. Morton Award for his "seminal contributions to semiconductor process and device modeling". 

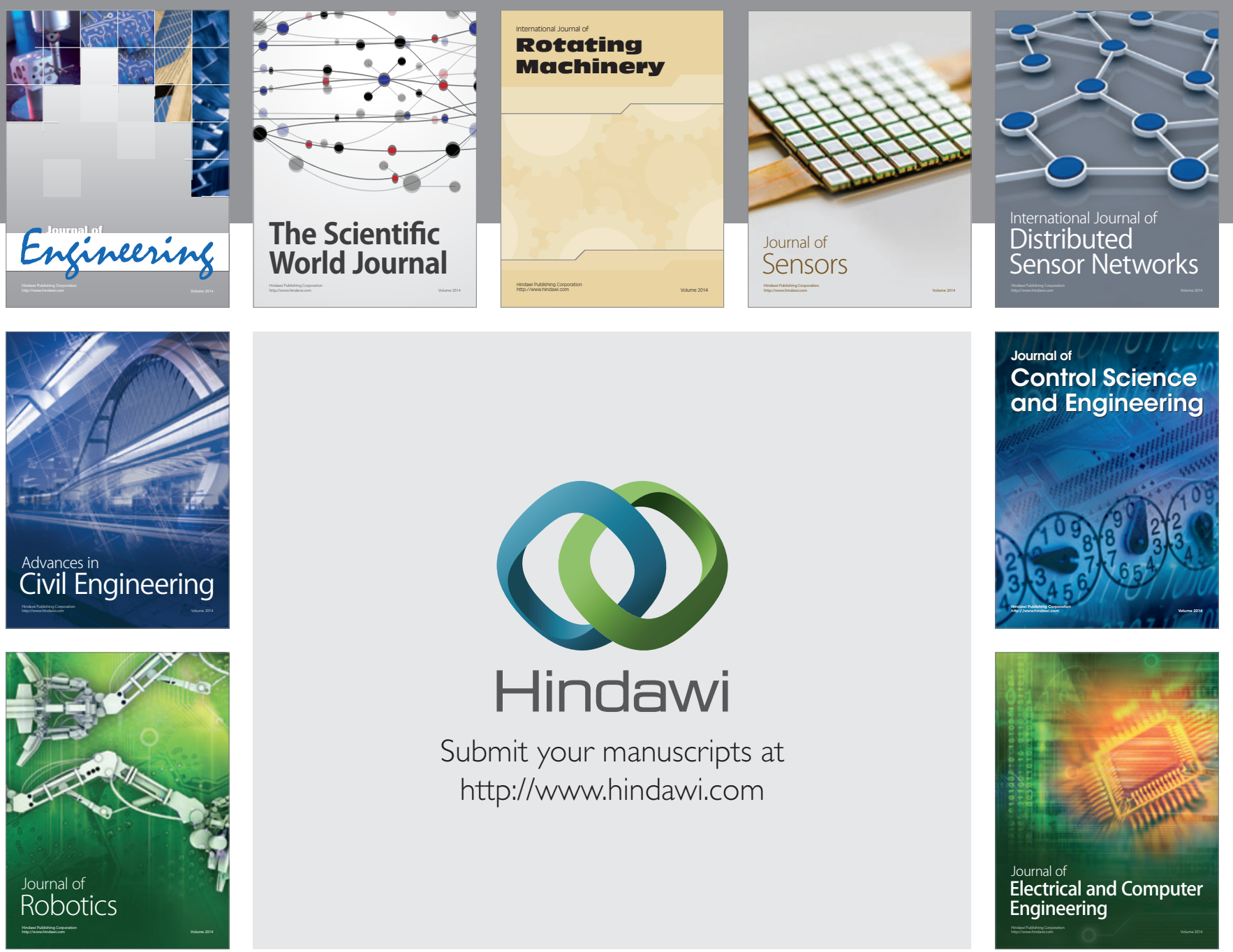

Submit your manuscripts at

http://www.hindawi.com
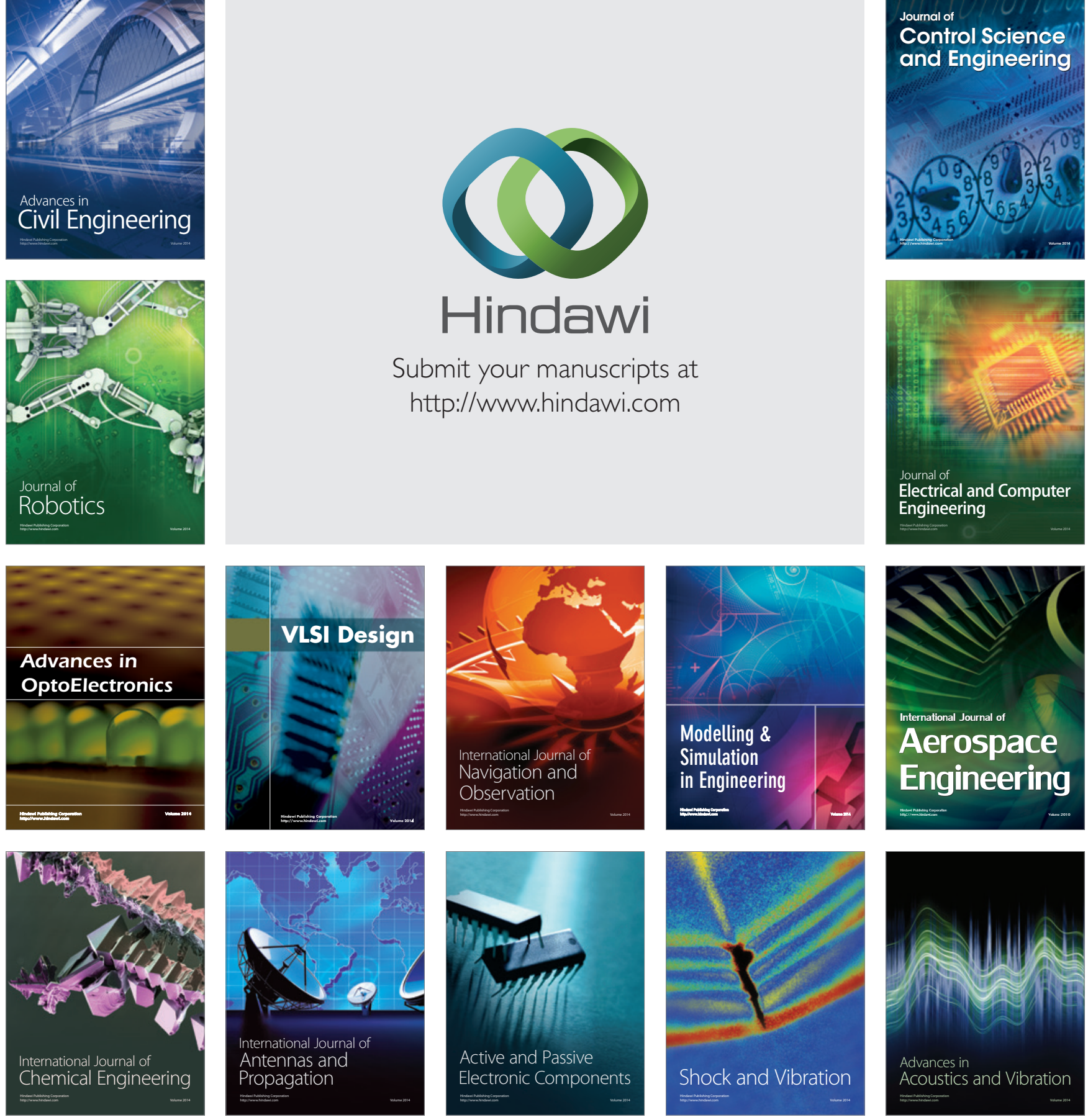